\title{
Altération du minerai de fer dans les mines abandonnées de Lorraine
}

\section{GRGIC \\ F. HOMAND}

Laboratoire Environnement, Géomécanique et Ouvrages École nationale supérieure de géologie de Nancy rue, du Doyen-Marcel-Roubault BP 40

54501 Vandceuvre-lèsNancy Dragan.Grgic@ensg.inplnancy.fr Françoise.Homand@ensg.in pl-nancy.fr

\section{G. DAGALLIER}

UMR 7566

Géologie et Gestion

des Ressources minérales

et énergétiques

CREGU, Nancy

Guy.Dagallier@ensg.inplnancy.fr
Une étude de l'évolution minéralogique du minerai de fer de Lorraine formant les piliers abandonnés des anciens travaux a été réalisée pour mieux comprendire le mécanisme des effondrements miniers. Des échantillons ont été prélevés dans une zone sans travaux miniers, au cœur de certains piliers et au bord de piliers dont la date d'exposition à l'atmosphère minière est connue. Les échantillons ont été analysés au MEB, à la microsonde et au diffrractomètre à rayons $\mathrm{X}$. Il en résulte que la minéralogie et la microstructure du minerai de fer exploité évoluent avec le temps. Cette évolution, d'origine anthropique, diffère de l'évolution diagénétique. Elle correspond à une première phase d'altération du ciment inter-oolitique (carbonates de fer et phyllosilicates ferrifères) suivie par une seconde phase de néoformation de phyllosilicates ferrifères puis d'un dépôt d'oxydes de fer. Une étude de microbiologie a montré l'influence des bactéries sur ces phénomènes. L'influence des conditions physico-chimiques environnantes a également été discutée. Ces modifications microscopiques se traduisent à l'échelle macroscopique par une diminution de la cohésion et donc de la résistance de la roche, et influent donc sur la stabilité à long terme des piliers abandonnés.

Mots-clés : mines de fer, effondrements, minéralogie, altération, oxydation, vieillissement, bactéries.

\section{Weathering of iron ore in Lorraine abandoned mines}

In order to explain mining collapses, which currently occur in Lorraine, a study on iron ore ageing constituting abandoned pillars of old workings has been performed. Samples have been taken in an area without mining workings, in the centre of some pillars and at the edge of some pillars whose date of exposition to the mining atmosphere is known for each one. Samples have been analysed with Scanning Electron Microscope, electronic microprobe and X-ray diffractometer. It results that iron ore mineralogy and micro-structure evolve with time in working zones. This evolution, of anthropogenic origin, differs from diagenetic evolution. It corresponds to a first stage of interoolitic cement (iron carbonates and iron phyllosilicates) weathering followed by a second stage of iron phyllosilicates and iron oxides crust neoformation. Microbiological analysis have shown the influence of bacteria on these phenomena. The influence of surrounding physicochemical conditions has also been discussed. These microscopic modifications appear on a macroscopic scale as a decrease of iron ore cohesion and maximal strength, and affect on the long-term behaviour of abandoned pillars.

Key words : iron mines, collapses, mineralogy, weathering. oxidation, ageing, bacteria. 


\section{Introduction}

Pendant près d'un siècle, jusqu'en 1980, l'exploitation du minerai de fer en Lorraine a généralement été conduite selon la méthode des chambres et piliers. La majorité des piliers résiduels a été foudroyée sous les zones non habitées, mais il subsiste des piliers sous certaines localités, constituant ainsi des stots de protection. Ces stots sont abandonnés depuis près de 100 ans pour certains. Des effondrements se sont produits dans ces zones habitées (Grgic, 2001) en phase d'exploitation. Puis, la fermeture des exploitations a entrainé l'arrêt récent des pompages d'exhaure et donc la remontée de la nappe aquifère. Quelques effondrements se sont produits au cours de cette phase.

Aussi, un important programme de recherche a-t-il été mis en place pour tenter de résoudre les problèmes qui se posent en ce qui concerne la sécurité en surface et donc l'aménagement du territoire. Ce programme, concu par le GISOS (Groupement de recherche sur l'Impact et la Sécurité des Ouvrages Souterrains), comprend des études sur le vieillissement des roches, le comportement différé, la surveillance par capteurs acoustiques, la géochimie des eaux, l'estimation et la hiérarchisation des risques. Les recherches menées dans le cadre du GISOS ont pour but de répondre aux interrogations suivantes : faut-il continuer à ennoyer les galeries? Peut-on encore construire au-dessus d'anciennes exploitations, comment surveiller et quels sont les critères d'alerte? Cet article porte sur l'un des points permettant d'éclairer le problème: le vieillissement du minerai de fer constituant les piliers abandonnés et exposés à l'atmosphère minière depuis le début des exploitations.

La formation ferrifère lorraine peut atteindre une puissance de 60 mètres et les mines les plus profondes sont à 250 mètres sous la surface. Cette formation com- porte une succession de couches ferrifères, le minerai exploité, et de couches moins riches en fer, les « marnes intercalaires '), constituées d'une alternance d'argilites, de siltites et de carbonates. La faible teneur en fer du faciès minéralisé ( 30 à $35 \%$ ) a valu au minerai de fer lorrain le qualificatif de «minette ».

Les différentes couches de minerai sont désignées par une couleur (successivement du sommet à la base du gisement : rouge, jaune sauvage, jaune principale, grise, brune, noire et verte), termes de mineurs, et chaque couche a des épaisseurs et des teneurs en fer variées. Le minerai de fer est donc très hétérogène et, comme l'a déjà noté J.-L. Pineau (1978), cette hétérogénéité est de dimension variable dans l'espace. Ce caractère est très important car l'hétérogénéité va se traduire par des variations du comportement mécanique et par une réactivité différente aux conditions physico-chimiques environnantes, conduisant au vieillissement.

Chaque couche de minerai s'inscrit dans une séquence de trois termes lithologiques élaborée en milieu littoral (Bubenicek, 1961, Teyssen, 1989) comportant de haut en bas : un niveau calcaire coquillier grossier (biocalcirudite) formant le crassin, le minerai constitué principalement d'une ferri-arénite, un intercalaire de siltite argileuse et carbonatée.

Habituellement la ferri-arénite d'un minerai sain comporte une fraction clastique riche en oolites jointives faites d'un oxy-hydroxyde de fer, la goethite. Cet ensemble à grains ovoïdes ou sphériques (Fig. 1) est cimenté par un mélange de calcite, de sidérite et de phyl. losilicates en proportions variées, Les spectres d'analyse réalisés en parallèle avec l'observation au microscope électronique à balayage montrent que les oolites de goethite comportent en moyenne environ $11 \%$ d' $\mathrm{Al}_{2} \mathrm{O}_{2}, 9 \%$ de $\mathrm{SiO}_{2}$ ainsi que 3 à $7 \%$ de $\mathrm{P}_{2} \mathrm{O}_{5}$ (Grgic, 2001).

Les cortex oolitiques montrent des couches concentriques (Fig. 2) dont la formation serait liée à des processus biochimiques (Burkhalter, 1995 ; Konhauser,

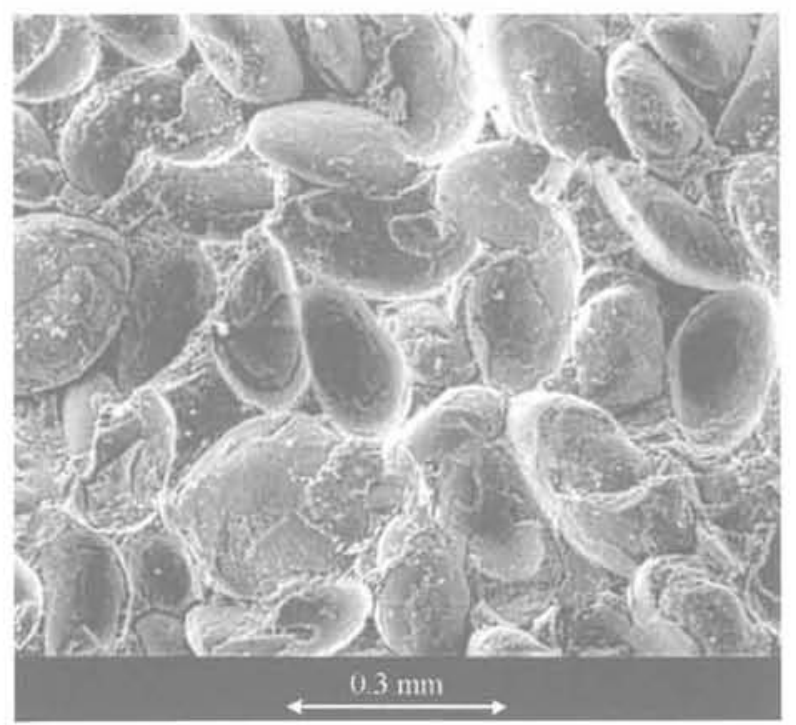

AG.1 Surface de cassure typique du minerai de fer oolitique (observation au MEB). Typical crack surface of oolitic iron ore (MEB observation)

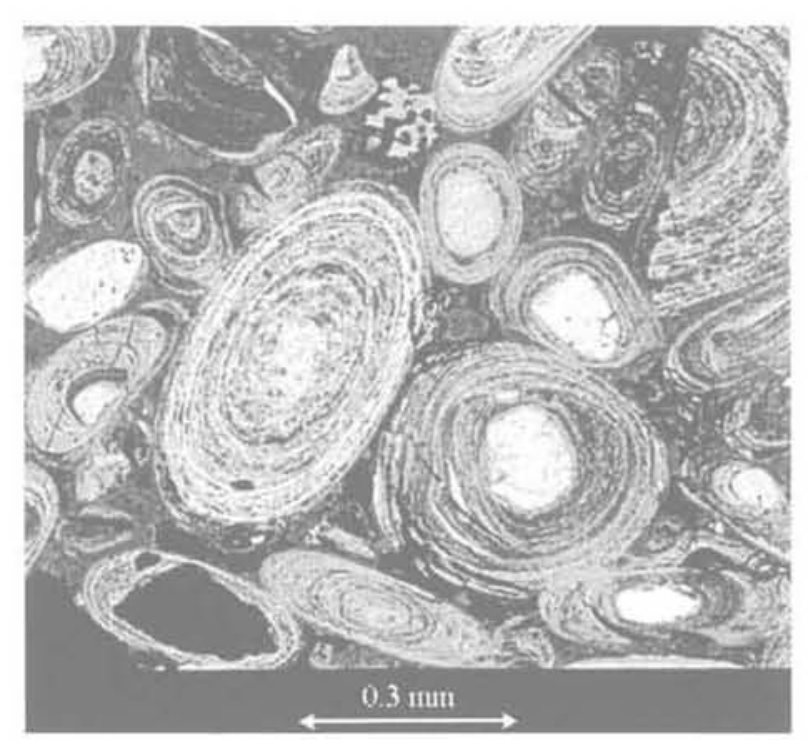

FG. 2 Organisation texturale typique du minerai de fer oolitique. Les cortex sont formés de couches concentriques de goethite (observation MEB sur section polie).

Example of iron ore textural organisation. Cortex are made by concentric lavers of goethite (MEB observation on polished section). 
1998) ; ils renferment parfois quelques phyllites argileuses. D'autres éléments détritiques, fragments carbonatés de coquilles, quartz, débris phosphatés d'os, s'y associent (Bubenicek, 1961). Le ciment de sidérite renferme souvent du calcium et du magnésium en remplacements isomorphiques du fer (sidérite ankéritique). Un phyllosilicate diagénétique, de teinte verte, y est généralement associé ; il s'agit d'une espèce très ferrifère, considérée depuis toujours comme étant de la chamosite (Bubenicek, 1970). Celleci forme souvent un liséré autour des fragments détritiques ou remplit, avec ou sans sidérite associée, la totalité de l'espace inter-oolitique (Dagallier, 1997). Elle comporte en général une proportion de $\mathrm{SiO}_{2}$ (près de $30 \%$ ) 1,5 à 2 fois plus élevée que celle d' $\mathrm{Al}_{2} \mathrm{O}_{3}$.

Le faciès le plus commun des couches minéralisées est la ferri-arénite, riche en fer et en oolites. Le minerai ferri-arénite renferme souvent des passès de biocalcarénite. Le minerai de fer se présente donc sous la forme d'une superposition de faisceaux de ces deux termes ferrugineux et carbonaté, intimement associés et à litage oblique.

\section{Procédure expérimentale}

La plupart des études sur le vieillissement ont été réalisées sur des matériaux de construction, tels le béton, et le vieillissement est en général défini comme une modification des caractéristiques mécaniques. Dans le cadre des mines de fer abandonnées de Lorraine, l'étude sur le vieillissement du minerai de fer prend en compte l'évolution des caractéristiques physiques et minéralogiques induites par son exploitation. L'altération se produit, en effet, parce que les minéraux constitutifs de la roche ne sont plus en équilibre thermodynamique avec les conditions environnantes. On s'intéresse donc au processus d'altération chimique. L'altération chimique transforme la composition initiale de la roche par mise en solution ou par précipitation d'éléments. Le vecteur dominant de l'altération chimique est donc bien évidemment l'eaut.

La comparaison des analyses (MEB, microsonde, diffraction $\mathrm{X}$, dosage des carbonates par analyse chimique, analyses bactériologiques) réalisées sur du minerai de fer vieilli par rapport à celles réalisées sur du minerai de fer sain, prélevé dans une zone non exploitẻe, a pour but de caractériser l'évolution anthropique de la roche. Afin de déterminer la nature de l'évolution du minerai de fer, l'étude doit donc mettre en évidence :

- l'état actuel de l'évolution diagénétique du minerai de fer «sain ») ;

- les caractéristiques physico-chimiques et minéralogiques du vieillissement anthropique.

En d'autres termes, il s'agit de déterminer:

- la nature des équilibres minéraux actuels et passés ; - la nature des transformations minéralogiques et microstructurales ainsi que celle des néoformations :

- l'influence de l'exploitation minière sur tous ces phénomènes.

Nous avons caractérisé les deux faciès (ferri-arénite et bio-calcarénite) du minerai de fer de la couche grise, la plus exploitée du gisement ferrifère lorrain. Les échantillons analysés ont été prélevés dans différents types de sondages carottés. Des sondages verticaux (Fig. 3) ont été réalisés, d'une part, depuis la surface dans une zone éloignée de toute exploitation (Malavillers) et, d'autre part, dans une zone exploitée où ils traversent le coeur des piliers sur toute leur hauteur (Jœuf).

Les échantillons provenant de la zone vierge doivent permettre de caractériser l'état minéralogique actuel du minerai de fer, considéré comme sain, et d'en différencier l'évolution anthropique que l'on va désigner par le terme de vieillissement. Pour étudier le vieillissement, des sondages horizontaux ont été réalisés dans plusieurs piliers (jusqu'à $80 \mathrm{~cm}$ de profondeur) dont la date d'exposition à l'atmosphère minière est connue. Ces échantillons ont été prélevés dans le stot de 'Tressange aux dates d'exploitations suivantes : 1990 . 1970 , vers 1930, et entre 1900 et 1910 .

Afin de qualifier et d'expliquer les évolutions minéralogiques du minerai de fer dans le contexte des mines de fer abandonnées, les techniques d'analyse suivantes ont été utilisées:

- observations au microscope électronique à balayage (MEB) sur fragments bruts, couplées à des analyses par spectrométrie par dispersion d'énergie (EDS) permettant d'obtenir la composition chimique semi-quantitative des minéraux:
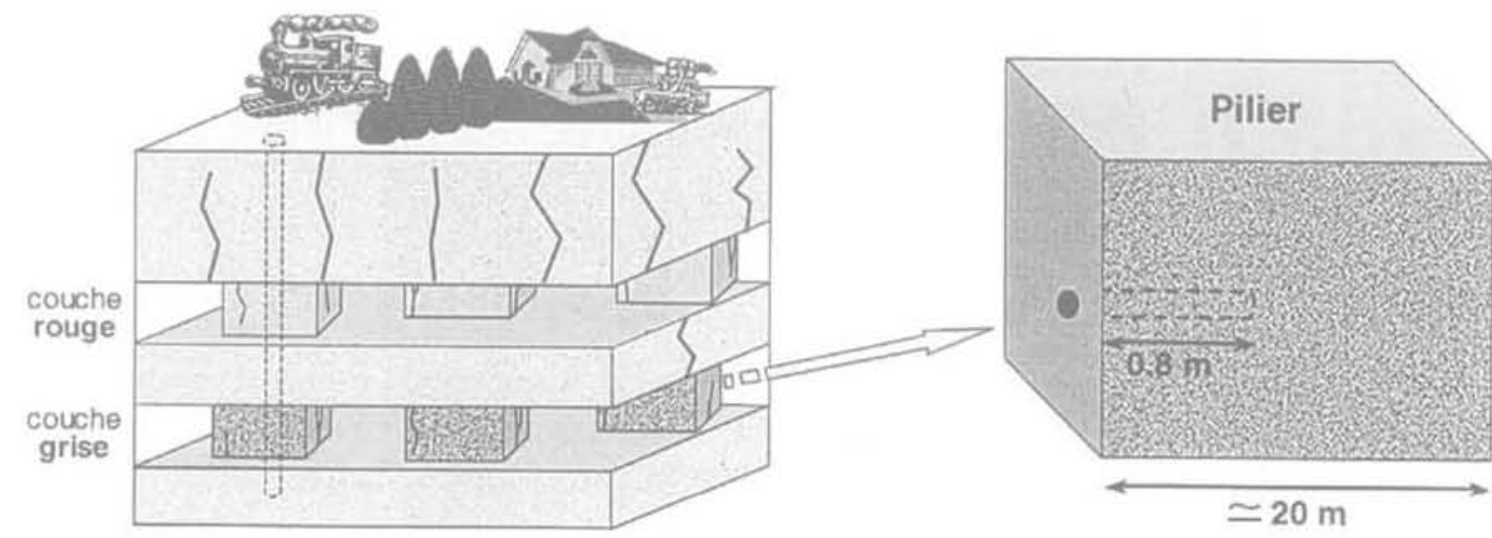

7G. 3 Sondages verticaux et horizontaux réalisés dans la formation ferrifère. Vertical and horizontal core drillings performed in iron formation. 
- analyses à la microsonde électronique sur lames minces et sections polies. Cette technique est utilisée en complément du MEB et de l'EDS pour déterminer la composition chimique précise des minéraux observés à partir de spectres d'analyse quantitatifs ;

- analyses par diffractométrie X. Ces analyses permettent d'identifier de manière précise toutes les phases minérales cristallisées présentes dans un échantillon :

- analyses microbiologiques. Ces analyses, qui sont encore en cours, sont réalisées afin d'étudier l'influence des bactéries sur les phénomènes de vieillissement.

\section{3}

\section{Résultats des analyses minéralogiques}

\section{1}

\section{Observations et analyses au MEB}

Un échantillon de minerai de fer sain de la couche grise a été prélevé dans une zone vierge de toute exploitation (Malavillers). Les observations au MEB montrent que le minerai de fer, dont une vue d'ensemble est donnée sur la figure 4, n'est pas altéré ; les oolites sont saines, il n'y a pas de microfissures et le ciment, riche en phyllosilicates ferrifères et en carbonates, est abondant et bien cristallisé. Ces observations concernent donc l'état actuel du minerai de fer, état qui prend en compte l'évolution diagénétique.

On observe dans les vides du ciment inter-oolitique (Fig. 5) des cristaux de phyllosilicates ferrifères dont une vue détaillée (Fig. 6) montre une forme de cristallisation géodique caractéristique, dite crêtée ou en rosette. Ces phyllosilicates semblent très « frais $)$ ou, du moins, plus frais que les phyllosilicates diagénétiques

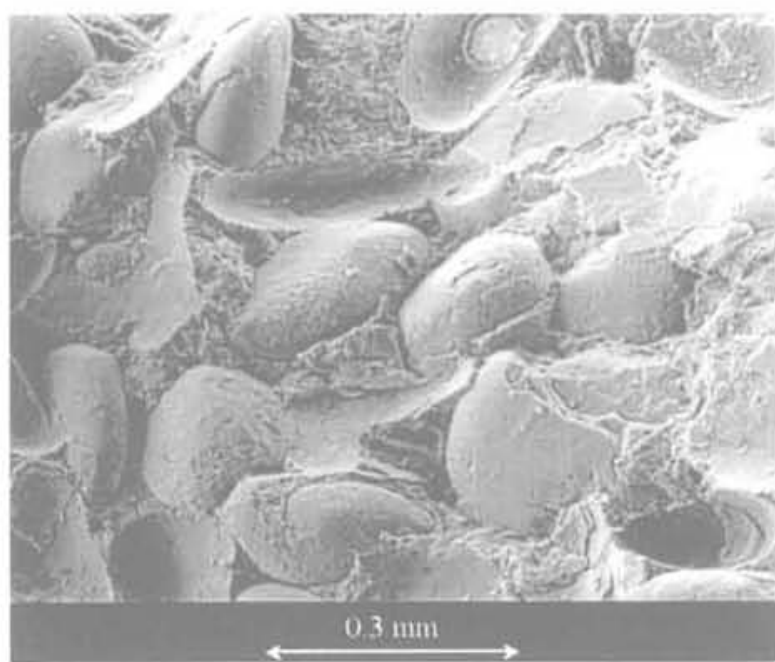

FIG.4 Minerai de fer sain prélevé dans une zone non exploitée (Malavillers), Les oolites d'oxyde sont bien cimentées par un assemblage de sidérite et de phyllosilicates ferrifères.

Healthy iron ore taken in an area without mining workings. Oxide oolites are wellcemented by an assembly of siderite and iron phyllosilicates.

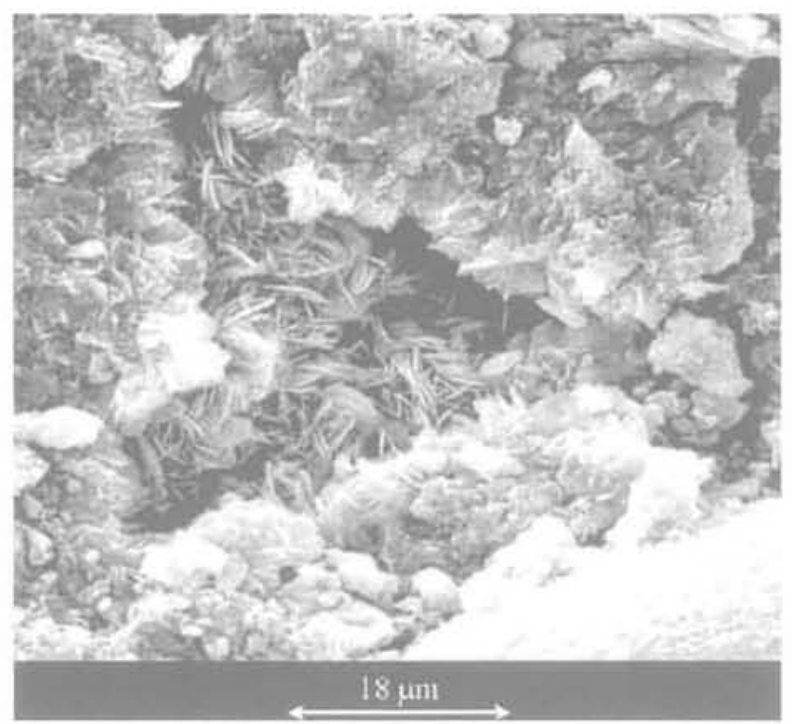

MG.5 Phyllosilicates ferrifères cristallisés sur les parois d'un vide ouvert dans les phyllosilicates diagénétiques. Minerai de fer sain.

Iron phyllosilicates crystallized on vacuum walls opened in diagenetic phyllosilicates. Healthy iron ore.

à structure cristalline floue situés à leur base. D'après les spectres EDS les compositions chimiques de ces cristaux sont proches.

Les échantillons prélevés au cœeur des piliers à partir des sondages carottés verticaux de Jœuf n'ont pu être datés, mais leur analyse peut apporter des informations complémentaires importantes quant à l'étude du vieillissement et de son étendue dans les piliers. Les carbonates de fer sont abondants et semblent à première vue peu altérés et bien cristallisés dans le ciment inter-oolitique (Fig. 7), Une observation plus fine (Fig. 8) montre que la sidérite commence localement à se dégrader avec un développement de fissures.

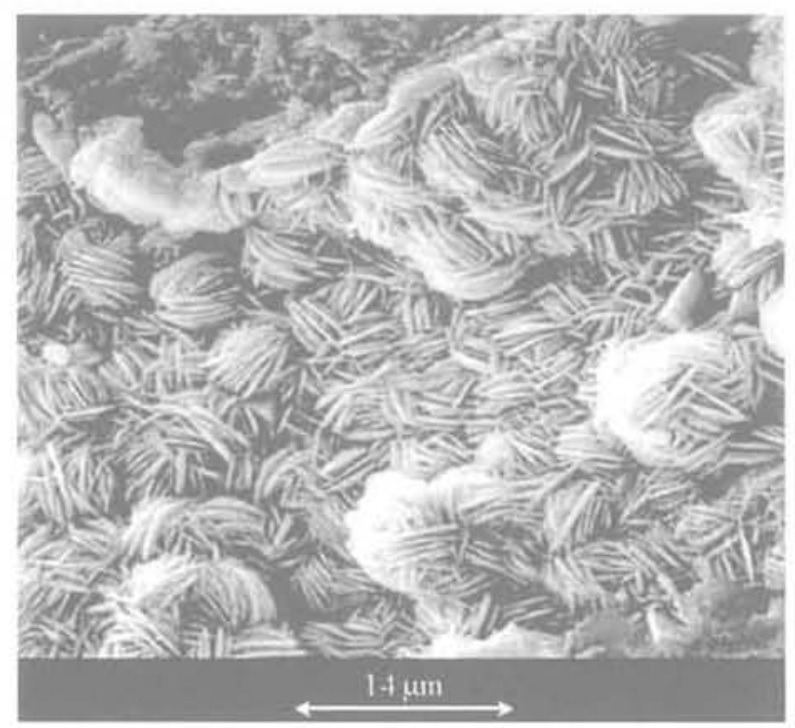

FIG.6 Détail des phyllosilicates ferrifères cristallisés en rosette et tapissant les parois d'un vide.

Detailed view of iron phyllosilicates crystallised in rosette form and lining walls of a vacuum. 


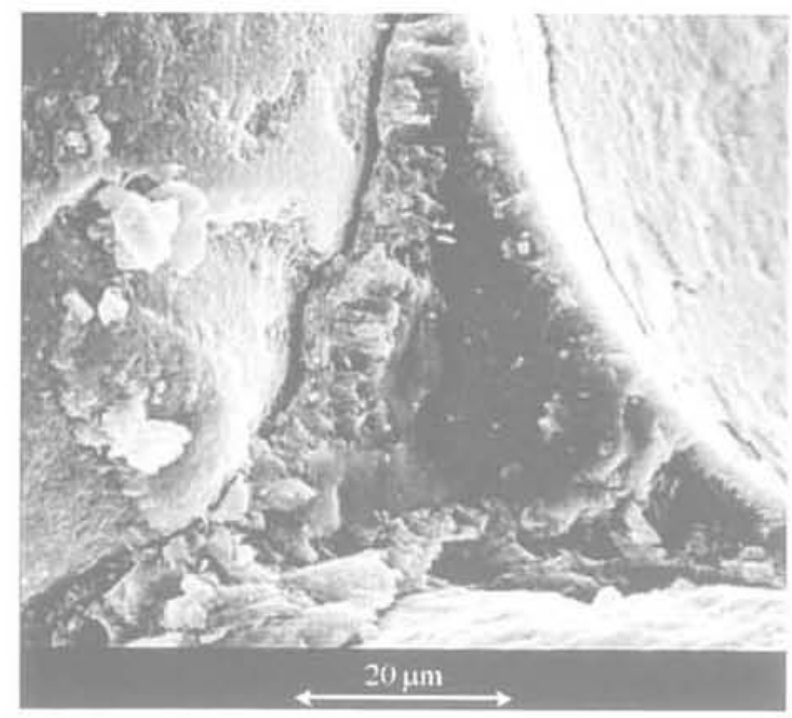

FIG.7 Carbonates de fer (sidérite) en bon état cimentant la porosité inter-oolitique.

Healthy iron carbonates (siderite) cementing the inter-oolitic porosity.

Le deuxième phénomène d'importance, observé sur ces échantillons prélevés au cceur des piliers, concerne l'évolution du ciment argileux. En effet, par rapport aux échantillons sains, les microcavités et les microfractures sont plus nombreuses et se remplissent de phyllosilicates ferrifères néoformés ou transformés (Fig. 9). Ces nouveaux minéraux prennent d'ailleurs de plus en plus d'importance et cela au détriment des phyllosilicates diagénétiques (Fig. 10) qui, à l'instar de la sidérite, commencent à s'altérer de façon importante. Ce phénomène de néoformation de phyllosilicates a également été observé dans la couche rouge. Dans certains cas, les contacts entre les oolites deviennent directs et certaines oolites s'écrasent mutuellement (Fig. 11). La structure intra-oolitique est endommagée et le cortex se desquame. Ainsi découvert, il tend à s'altérer et perd sa cohésion interne.

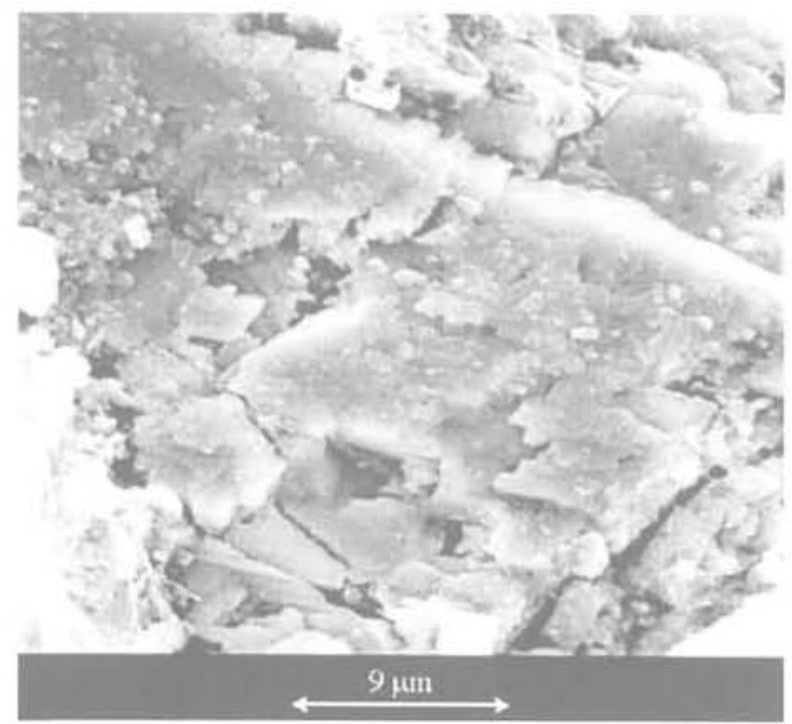

FIG. 8 Traces d'altération et microfissures dans la sidérite inter-oolitique.

Weathering and microcracks in inter-oolitic siderite.

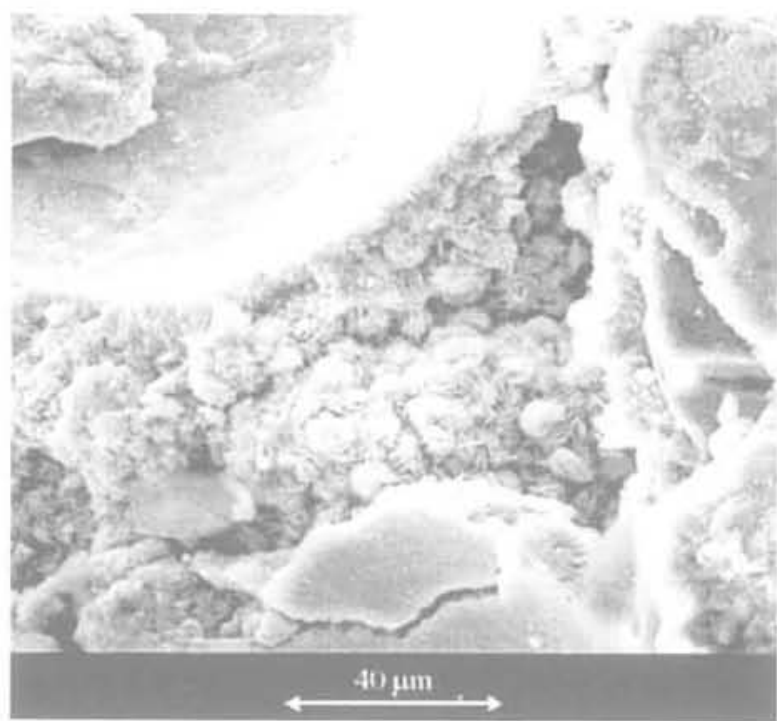

\#iG. 9 Néoformation de phyllosilicates ferrifères sur les parois d'un vide de l'espace interoolitique. Minerai de fer prélevé en çeur de pilier.

Iron phyllosilicates neoformation on vacuum walls of the inter-oolitic cement. Iron ore sample taken at the center of a pillar.

Sur les échantillons prélevés en bordure des piliers de la couche grise exposés à l'atmosphère minière depuis 10 à 30 ans, une altération semblable à la précédente s'observe et prend plus d'importance.

Les échantillons exposés à T'atmosphère minière depuis plus longtemps, entre 1900 et 1910 pour le premier et en 1930 pour le second, montrent dans un premier temps une raréfaction des phyllosilicates ferrifères. Ceux-ci, qu'ils soient d'origine diagénétique ou néoformés, semblent être totalement altérés. En outre, on constate la disparition presque totale des carbonates de fer (sidérite). Ces phénomènes marquent un état d'altération très avancé du minerai de fer. A l'évidence.

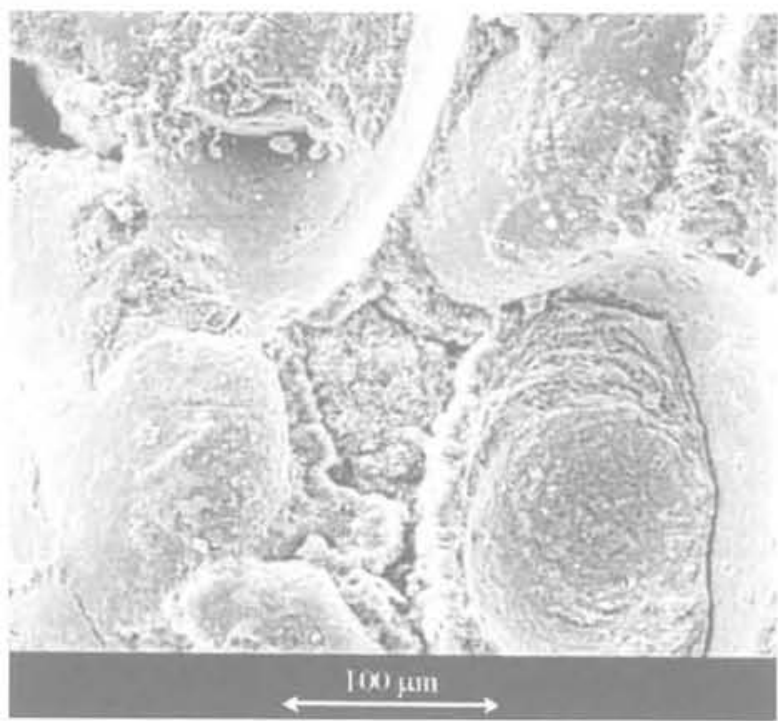

FG.10 Néoformation importante de phyllo. silicates et dégradation des phyllosilicates diagénétiques.

Important phyllosilicates neoformation and weathering of diagenetic phyllosilicates. 


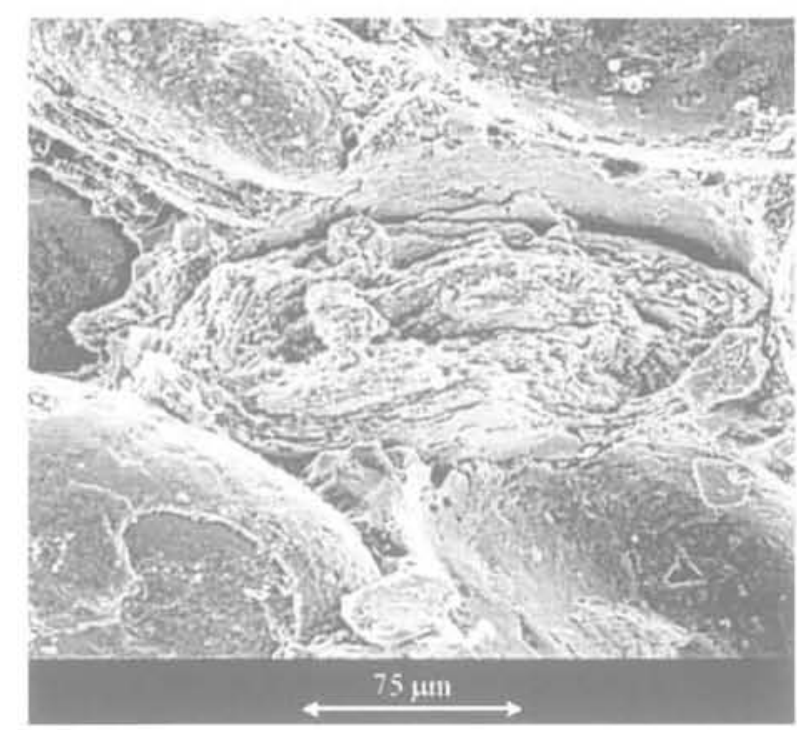

FG.11 Oolite écrasée entre d'autres oolites et dont le cortex se dégrade.

Oolite crushed between other ones and cortex weathering.

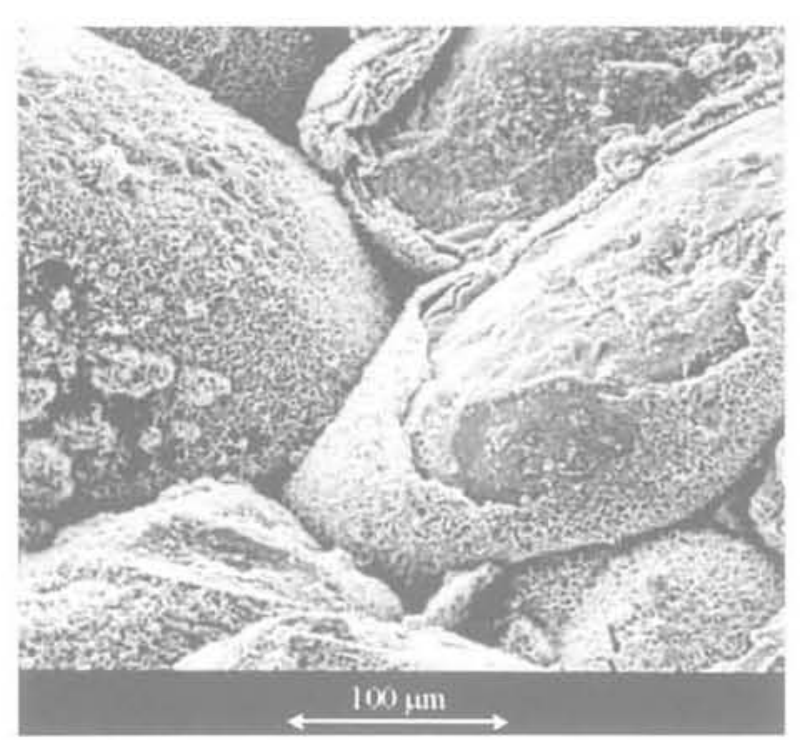

F16.12 Néoformation d'une croûte d'oxyde à la surface des oolites.

Échantillon de minerai exploité vers 1930.

Neoformation of an iron oxide crust on oolites. Iron ore sample exploited towards 1930

avant de tirer une conclusion, l'importante variabilité de la teneur relative en sidérite et, plus généralement. de la teneur en carbonates et en ciment inter-oolitique, nécessitera de réaliser ultérieurement des analysés à plus grande échelle.

Le dernier événement observé concerne la néoformation d'une croûte d'oxydes de fer (Figs. 12 et 13) dont la forme de cristallisation alvéolaire, dite en " nid d'abeille», est caractéristique. Cette croute, que l'on soupçonne ètre un dépòt résiduel dont la genèse serait liée à l'altération du ciment inter-oolitique, tapisse tous les supports encore existants. Ainsi, sous la forme d'une fine pellicule, elle enveloppe la surface des oolites ou recouvre les phyllosilicates du ciment. Sur la figure 14, les oxydes sont associés à des phyllosilicates néo- formés du ciment inter-oolitique. Bien que leur structure respective semble intimement liée, il semble bien que les oxydes soient de formation plus tardive. L'encroûtement représenté sur les figures 12 et 13 constitue la dernière étape visible de l'oxydation du ciment inter-oolitique,

Cette croûte d'oxydes ne peut cependant plus jouer le même rôle de ciment que jouaient la sidérite et les phyllosilicates avant dégradation ; les liens forts d'origine diagénétique, reliant initialement les oolites au ciment carbonaté et assurant ainsi la cohésion de l'ensemble, se sont rompus. L'adhérence est donc précaire et la croûte d'oxydes se détache alors facilement (Fig. 15). Les oolites, n'étant plus liées à ce qui reste du ciment inter-oolitique, se déchaussent fréquemment de leur emplacement originel (Fig. 15) et laissent alors

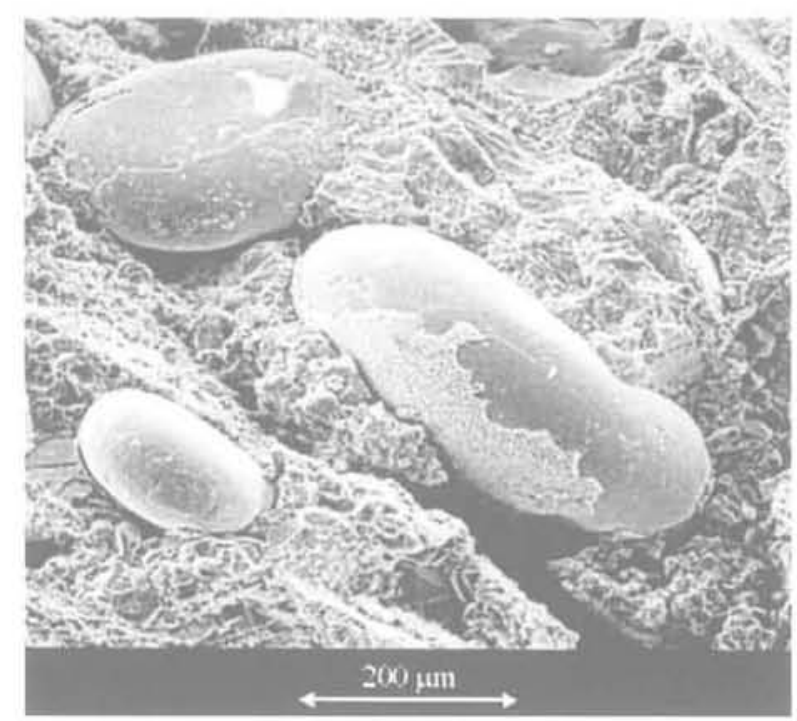

FG, 13 Néoformation d'une croûte d'oxyde à la surface des oolites.

Échantillon de minerai exploité vers 1900 1910.

Neoformation of an iron oxide crust on oolites Iron ore sample exploited towards 1900-1910.

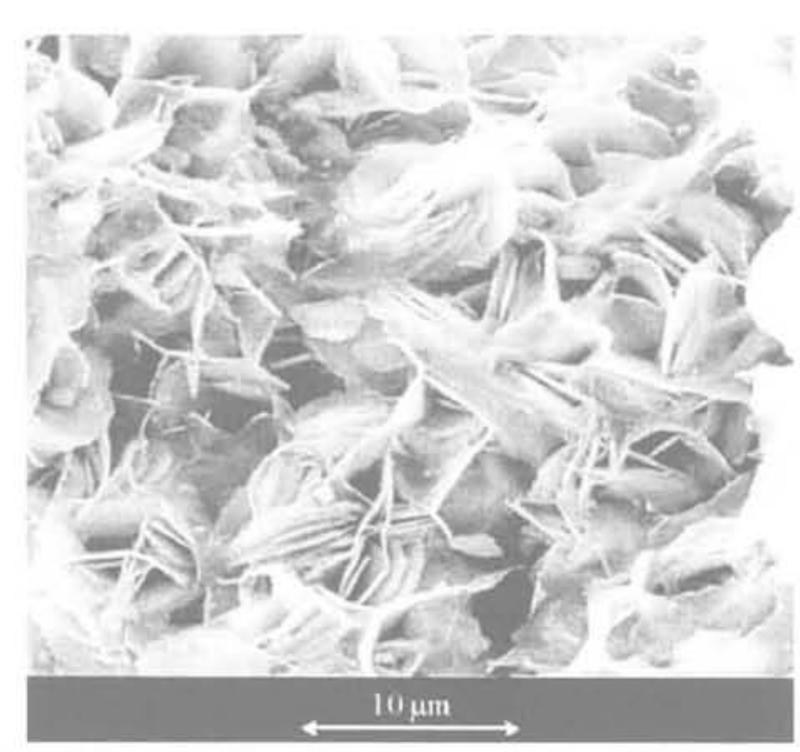

FIG.14 Feuillets de phyllosilicates recouverts d'une fine pellicule d'oxyde de fer.

Phyllosilicates leafs covered with a thin layer of iron oxide. 


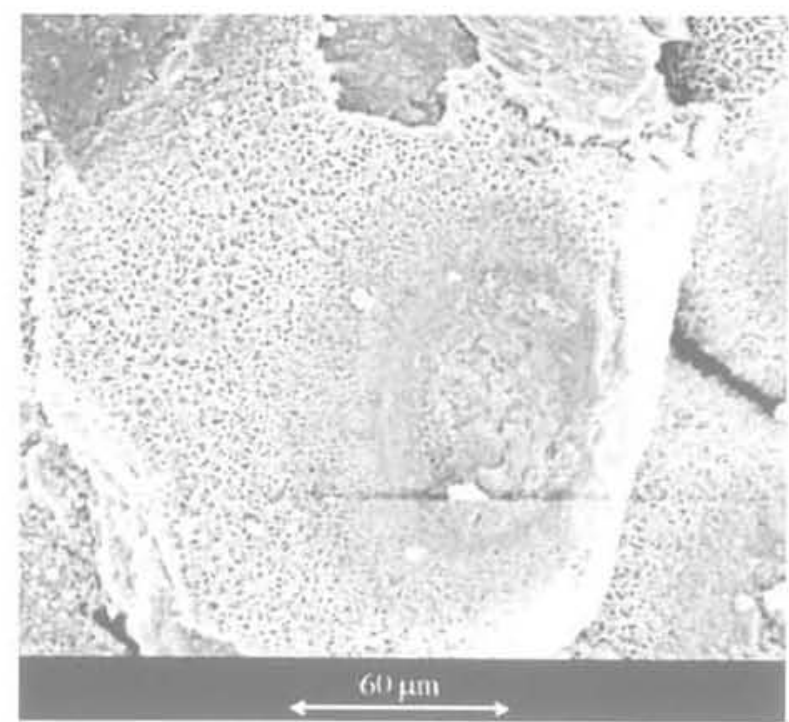

FG. 15 Oolites recouvertes par la croûte alvéolaire d'oxyde quí s'en détache par altération. Echantillon de minerai exploité vers 1930. Oolites wrapped by the honeycombed oxide crust that peels off the oolites by weathering. Iron ore sample exploited towards 1930.

apercevoir leur empreinte sur d'autres oolites en se "décollant » (Fig, 16).

Les oolites, recouvertes de cette couche de corrosion, présentent parfois un aspect altéré et leur cortex semble endommagé. Ceci est surtout valable pour les échantillons très ferrifères provenant du pilier exposé à l'atmosphère minière vers 1930 (Fig. 12), les échantillons du pilier exposé entre 1900 et 1910 étant plus carbonatés (Fig. 13) et, semble-t-il, moins sensibles à l'altération par oxydation. Peut-être faut-il y voir une relation entre l'aspect dégradé des cortex intra-oolitiques et la proportion initiale de sidérite présente dans le ciment inter-oolítique et susceptible de disparaître par altération.

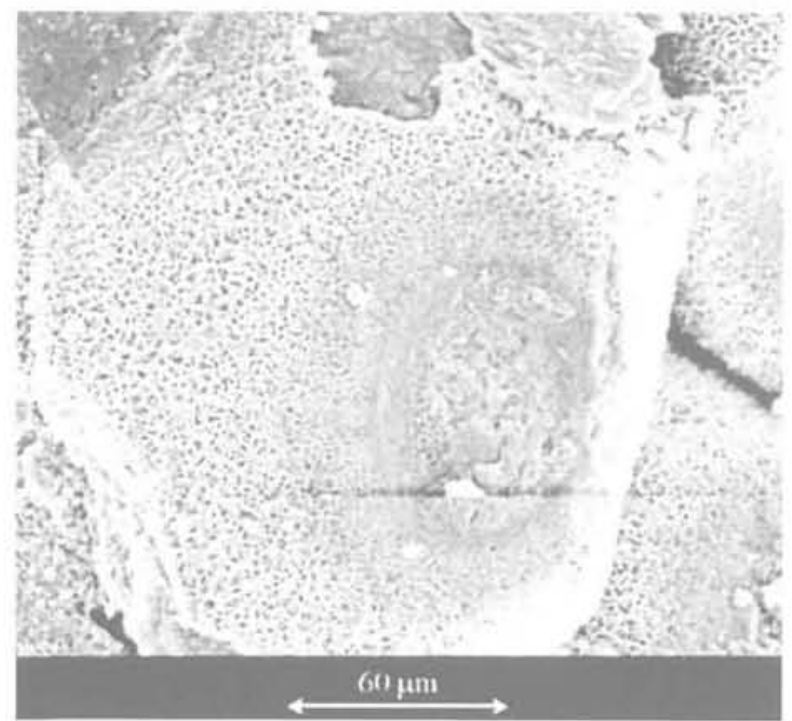

Fic.16 Empreinte d'une oolite décollée en surface d'une autre oolite.

Échantillon de minerai exploité vers 1930. Print of an oolite detached on the surface from other one. Iron ore sample exploited towards 1930.
Toutes ces observations indiquent donc que la roche soumise à l'atmosphère de la mine tend à perdre sa cohésion inter-oolitique et intra-oolitique.

TABLEAUI Composition chimique des mínéraux observés au MEB. Spectres d'analyse EDS semi-quantitatifs.

Chemical composition of the minerals observed with SEM. Semi-quantitative EDS absorption spectra.

\begin{tabular}{l|c|c|c|c|c}
\hline \% oxyde & $\mathrm{SiO}_{2}$ & $\mathrm{AL}_{2} \mathrm{O}_{3}$ & $\mathrm{MgO}$ & $\mathrm{P}_{2} \mathrm{O}_{3}$ & $\mathrm{FeO}$ (tot.) \\
\hline $\begin{array}{l}\text { Cortex oolithique } \\
\text { (goethite) }\end{array}$ & 9 & 11 & 3 & 3 à 7 & 70 \\
\hline $\begin{array}{l}\text { Phyllosilicate } \\
\text { diagénétique }\end{array}$ & 32 & 18 & 6 & n.s. & 43 \\
\hline $\begin{array}{l}\text { Phyllosilicate } \\
\text { néo-cristallisé } \\
\text { état altéré }\end{array}$ & 28 & 20 & 5 & n.s. & 46 \\
\hline $\begin{array}{l}\text { Croúte d'oxydes } \\
\text { néoformée }\end{array}$ & 5 & 9 & 5 & n.s. & 76 \\
\hline
\end{tabular}

n.S: : non significatif.

\section{2}

\section{Identification des minéraux observés}

La composition chimique des minéraux observés est présentée dans le tableau l. Les valeurs sont obtenues à partir des spectres d'analyse EDS semi-quantitatifs, sur des fragments bruts de roche. Les compositions chimiques qui correspondent à des analyses ponctuelles sont donc approchées et sont présentées sous forme de pourcentages d'oxydes. Elles sont au moins au nombre de 5 pour chaque minéral étudié et les chiffres présentés correspondent à une moyenne arithmétique.

Ces résultats montrent la présence en quantité non négligeable ( 3 à $7 \%$ ) de phosphore dans la structure de la goethite. La croüte néoformée a une composition relativement proche de celle de la goethite du cortex, à la différence près que la proportion des autres constituants que le fer (silice, alumine...) est plus faible. Il pourrait s'agir soit d'hématite, soit d'une goethite plus pure que celle du cortex, soit d'un hydroxyde de fer. Dans le ciment inter-oolitique, les phyllosilicates néoformés ou transformés dans les échantillons vieillis sont constitués en moyenne de $20 \% \mathrm{~d}^{\prime} \mathrm{Al}_{2} \mathrm{O}_{3}, 28 \%$ de $\mathrm{SiO}_{\text {, et }} 46 \%$ de $\mathrm{FeO}$, et leur composition chimique s'avère donc relativement différente (diminution de la quantité de $\mathrm{SiO}_{2}$ ) de celle des phyllosilicates diagénétiques. Dans toús les cas, il s'agit d'espèces très ferrifères.

Les spectres d'analyse quantitative obtenus par la microsonde électronique permettent de préciser la composition de certains minéraux analysés au MEB. Ils sont obtenus sur des lames minces, support pour lequel le bilan des teneurs boucle correctement. Pour la croûte d'oxydes néoformée sur les échantillons de minerai de fer vieilli, on a obtenu la composition chimique suivante (moyenne sur 7 mesures) : $0: 28,3 \%$; $\mathrm{Al}: 1,4 \% ; \mathrm{Si}: 2,4 \%$ et $\mathrm{Fe}: 56,6 \%$. Les impuretés présentes sont en faibles quantités.

Des analyses diffractométriques aux rayons $\mathrm{X}$ ont été réalisées sur le minerai de fer sain et vieilli. Il n'y a pas de différence fondamentale entre ces différentes analyses. Il est très difficile de faire une distinction entre 
les différents oxydes de fer, comme l'hématite et la goethite, à partir d'un diffractogramme. Il existe cependant une méthode, basée sur la spectroscopie Mössbauer, le permettant (Loef, 2000). La principale information concerne l'absence de la raie caractéristique à $14 \AA$ de la chamosite. Les phyllosilicates observés au MEB correspondent donc à de la berthiérine. Ce résultat, qui est pour le moins surprenant dans le sens où Bubenicek (1970) affirme que le minerai de fer est formé d'une chiorite ferrifère de type chamosite, demande à être vérifié par des analyses plus nombreuses et plus variées (prélèvements à différents endroits du gisement). Par ailleurs, les raies caractéristiques de la sidérite, dont la principale à $2,79 \AA$, sont introuvables dans le diffractogramme réalisé sur l'échantillon de minerai de fer le plus altéré (exposé en 1930), mais bien présentes dans les autres diffractogrammes réalisés sur les échantillons non altérés (en cour de pilier ou récemment exploités). Ce fait confirme, en attendant un plus grand nombre de mesures qui permettraient de s'affranchir des hétérogénéités du matériau, que la sidérite contenue dans le ciment inter-oolitique s'altère en vieillissant et tend donc à disparaitre.

\section{4}

\section{Modèle phénoménologique de vieillissement}

A partir de toutes les analyses et observations précédentes, nous proposons un modèle phénoménologique d'évolution dans le temps, et en plusieurs étapes, pour le minerai de fer constituant les piliers abandonnés. Les évolutions d'origine anthropique dans les piliers de minerai seront désignées par les termes transformations et néoformations. Les produits de transformation sont, pour le minerai de fer, les phyllosilicates néo-cristallisés en rosette sur les phyllosilicates diagénétiques, Les produits de néoformation cristallisent à partir des ions en solution. Les oxydes ou hydroxydes de fer formant la croûte néoformée ainsi que les phyllosilicates en rosette remplissant les vides, font partie des minéraux ainsi élaborés.

\section{État actuel de l'évolution diagénétique du minerai de fer}

Le minerai de fer est très poreux (de 20 à $35 \%$ ) et assez perméable (environ $10^{18} \mathrm{~m}^{2}$ de perméabilité de matrice). Cette roche correspond donc à un milieu chimiquement très réactif. Le minerai de fer est riche en oxydes et dans les zones non exploitées, il est en équilibre chimique avec l'eau interstitielle depuis des millions d'années. Après formation et enfouissement, les conditions chimiques sont celles d'un milieu réducteur et ont permis la formation d'un ciment riche en sidérite et en phyllosilicate ferrifère, qui sont des minéraux oú le fer est à l'état réduit au cours de la diagenèse précoce. L'analyse d'échantillons prélevés dans une zone éloignée de toute exploitation minière nous a permis d'identifier l'état actuel de l'évolution diagénétique du minerai de fer, état qui va servir de référence aux observations suivantes sur le minerai vieilli. En l'absence de conditions chimiques extérieures oxy- dantes, les berthiérines et les carbonates de fer évoluent dans un milieu propice à leur stabilité.

\section{2}

\section{Phase transitoire de l'exploitation}

Les travaux miniers vont modifier de manière fondamentale les conditions chimiques puisque le minerai de fer va se trouver dans des conditions oxydantes. Le milieu est, à l'origine, réciucteur et l'exposition des piliers à l'atmosphère humide régnant dans les mines abandonnées est propice au développement de réactions minéralogiques d'oxydation. Ainsi, l'exploitation va ètre à l'origine de transformations de certains minéraux du minerai de fer et de modifications microstructurales. Tous ces phénomènes sont donc d'origine anthropique et ils ont été observés sur du minerai de fer situé en borcure et, pour certains, à l'intérieur des piliers.

Les traçages et dépilages successifs en cours d'exploitation, ainsi que le phénomène de redistribution des contraintes, vont endommager les piliers résiduels. La conséquence est le développement de fissures de l'échelle de la microstructure à celle du pilier. Cette phase transitoire d'exploitation est le point de départ du vieillissement anthropique du minerai de fer puisqu'elle va initialiser et favoriser les étapes successives de l'évolution minéralogique dans les piliers. La présence de grandes fractures va amplifier le vieillissement à l'intérieur des piliers, et surtout à proximité des fronts de taille, séparant les stots de protection des zones antérieurement foudrovées, où les roches sont très fracturées et très lessivées. Le lessivage des roches est, en effet, une condition très favorable à l'amplification des phénomènes d'altération.

\section{3}

\section{Phase progressive d'abandon des piliers}

Cette phase concerne dans un premier temps les phénomènes d'altération chimique des minéraux associés au lessivage et aux transferts hydriques dans les piliers. Cette phase d'altération concerne la berthiérine du ciment inter-oolitique. En effet, à plus ou moins longue échéance, les minéraux silicatés vont devenir instables et libérer une partie des éléments chimiques qu'ils renferment. La phase suivante va être la formation de nouveaux édifices cristallins stables dans les conditions de leur environnement. C'est à partir de cette étape que l'on peut réellement commencer à parler d'altération. Une altération chimique se développe dans une zone si le phénomène d'hydrolyse (action de l'acide carbonique sur des minéraux riches en cations) est favorisé (Cojan et Renard, 1997).

Le processus d'altération de la berthiérine s'installe dans tout le pilier. Il est accompagné par des transformations ou néoformations de ce même minéral. Les microfissures induites par l'exploitation renforcent ce phénomène puisque la création de vides est propice à la cristallisation de nouveaux minéraux. La composition chimique de ces nouvelles berthiérines est différente (diminution de la teneur en silice) de celle des berthiérines diagénétiques (du minerai sain ou vieilli). Ce type de phénomène, qui s'inscrit à la suite du processus diagénétique et qui a déjà été observé par ailleurs (Velde, 
1989), est lié au processus d'altération par hydrolyse. Les silicates en général, et les phyllosilicates en particulier, s'altèrent par hydrolyse de leurs constituants, au cours de laquelle les ions $\mathrm{O}^{2}$ de la charpente silicatée vont alors se lier préférentiellement aux ions $\mathrm{H}^{*}$ (de fort potentiel ionique) de la solution et libérer ainsi les cations $\mathrm{Al}^{3+}$ puis $\mathrm{Si}^{4+}$ dont le potentiel ionique est plus faible. Dans le cas d'une hydrolyse assez poussée, une partie importante de la silice ainsi que la totalité des cations basiques sont éliminés. Finalement, les feuillets altérés des berthiérines servent de base à la formation de nouveaux minéraux.

Cette première phase d'altération des piliers concerne également la sidérite du ciment inter-oolitique, La sidérite a effectivement la propriété de s'oxyder avec un rythme constant dans le temps. Ce phénomène est d'ailleurs identifié depuis longtemps dans le minerai de fer (Burchard, 1916), Dans des conditions physico-chimiques oxydantes (présence de $\mathrm{H}_{2} \mathrm{O}$ et $\mathrm{O}_{2}$ ) et lorsque le pH est proche de la neutralité, comme c'est le cas pour le minerai de fer qui est très riche en carbonates, l'oxydation du fer ferreux, libéré dans la solution aqueuse par dissolution de la sidérite, en fer ferrique est très rapide et est donnée par la réaction partielle suivante:

$$
\mathrm{Fe}^{2+}+1 / 4 \mathrm{O}_{2}+\mathrm{H}^{+} \rightarrow \mathrm{Fe}^{3+}+1 / 2 \mathrm{H}_{2} \mathrm{O}
$$

Le résultat de cette oxydation est l'altération de la sidérite dont la proportion dans le ciment inter-oolitique va diminuer avec le temps.

La phase d'abandon des piliers concerne ensuite les phénomènes de néoformation de minéraux. Ceux-ci dérivent de solutions concentrées en ions libérés des zones d'altération, et correspondent à une réorganisation complète du réseau cristallin. Les néoformations sont liées à un processus de dissolution-recristallisation.

En présence d'oxygène dissous ou adsorbé au niveau des micropores, les réactions d'oxydation précédentes ont lieu et le pH est localement acide. Mais en général, des valeurs relativement élevées de $\mathrm{pH}$ sont maintenues gràce au processus de neutralisation par des minéraux carbonatés tels que la calcite (Rose et al., 1998). Dans ces conditions, $\mathrm{Fe}^{3 *}$ (1) et $\mathrm{Al}^{3+}$ (issu de l'hydrolyse des phyllosilicates) précipitent sur place sous forme d'hydroxydes insolubles en s'unissant aux ions $\mathrm{OH}^{-}$(dissociation partielle de l'eau en $\mathrm{OH}^{-}$et $\mathrm{H}^{*}$ ). II peut alors se former des hydroxydes d'alumine et des hydroxydes de fer selon la réaction suivante:

$$
\left.\mathrm{Fe}^{3 *}+3 \mathrm{H}_{2} \mathrm{O} \rightarrow \mathrm{Fe}(\mathrm{OH})_{3} \text { (hydroxyde de fer }\right)+3 \mathrm{H}^{*}
$$

De la mème façon, la goethite foxy-hydroxyde de fer), produit d'altération le plus commun de la sidérite (Senkayi et al., 1986), peut cristalliser sur place. L'hématite (oxyde de fer), qui est également un produit d'altération de la sidérite (Deer et al., 1962), peut se former selon la réaction complète suivante:

$$
\begin{aligned}
2 \mathrm{Fe}^{2+}+4 \mathrm{HCO}_{3}^{-}+1 / 2 \mathrm{O}_{2}+4 \mathrm{H}_{2} \mathrm{O} \rightarrow \mathrm{Fe}_{2} \mathrm{O}_{3} \text { (hématite) } \\
+4 \mathrm{CO}_{2}+6 \mathrm{H}_{2} \mathrm{O}
\end{aligned}
$$

Ces réactions conduisent à la néoformation de la croûte oxydée observée dans les échantillons de minerai vieilli. Cette croûte est donc finalement un résidu cristallisé à partir des produits de dégradation du ciment inter-oolitique.

La réaction (1) nécessite la présence d'ions $\mathrm{H}^{*}$ dans la solution aqueuse, d'oủ la nécessité de passer par un milieu acide, du moins localement, étant donné la nature tamponnée du milieu. Cette acidité soutient et favorise l'oxydation de la sidérite et l'hydrolyse des phyllosilicates. La réaction de précipitation des hydroxydes (2) peut être une des sources de cette acidité. Il peut alors se créer un cycle oxydatif qui amplifie progressivement les réactions chimiques existantes. Par ailleurs, le volume de $\mathrm{CO}_{2}$ dissous dans l'eau contribue à la quantité d'ions $\mathrm{H}^{*}$ dísponibles dans la solution (4) et renforce le mécanisme de dissolution des minéraux carbonatés (sidérite) par l'eau.

$$
\mathrm{CO}_{2 \text { (iai) }}+\mathrm{H}_{2} \mathrm{O} \Leftrightarrow \mathrm{H}_{2} \mathrm{CO}_{3} \Leftrightarrow \mathrm{HCO}_{3}^{-}+\mathrm{H}^{+} \Leftrightarrow \mathrm{CO}_{3}^{2-}+2 \mathrm{H}^{+}
$$

\section{5}

\section{Conditions physico-chimiques et catalyseurs}

Avant exploitation, les fluides, pratiquement immobiles, réagissent avec les minéraux environnants dans un système clos et leur composition évolue en fonction des transformations diagénétiques. Dans les conditions de l'exploitation, les transferts hydriques dans les piliers deviennent importants puisque le milieu est soumis à un lessivage et à un aérage intenses. Les eaux karstiques, chimiquement déséquilibrées, s'infiltrent et vont accélérer tous les processus d'altération (dissolution). Les écoulements de fluides entraînent les produits de l'hydrolyse, notamment la silice, expliquant ainsi la composition des berthiérines transformées (ou néoformées). A l'intérieur des piliers, l'atmosphère n'est pas encore totalement oxydante et l'hydrolyse des phyllosilicates et l'oxydation de la sidérite devraient être limitées (la quantité d'ions $\mathrm{H}^{+}$est certainement plus faible). Ces phénomènes concernent l'état actuel du minerai de fer mais sont progressifs et évoluent donc constamment dans le temps. De plus, le vieillissement se propage au coeur des piliers selon un gradient d'intensité et au moins jusqu'à $80 \mathrm{~cm}$ de profondeur, c'est-à-dire la longueur maximale des sondages horizontaux réalisés jusqu'à présent.

Les propriétés physico-chimiques de l'eau interstitielle, notamment pH et Eh, ainsi que le potentiel d'oxydoréduction des différents équilibres minéraux, vont déterminer la nature des évolutions minéralogiques futures et la cinétique d'oxydation du fer ferreux. De plus, les réactions d'oxydoréduction et la réaction d'hydrolyse sont plus poussées dans des milieux bien drainés oủ la circulation des solutions favorise le renouvellement des ions $\mathrm{H}^{+}$, selon la réaction (4), ainsi que l'élimination des ions en solution. En outre, la quantité de $\mathrm{CO}_{2}$ dissous dans l'eau, et par conséquent la quantité d'ions $\mathrm{H}^{*}$ disponibles dans la solution aqueuse, est favorisée par la décomposition de matière organique présente dans le milieu.

Les conditions physico-chimiques locales ( $\mathrm{pH}$ et concentration d'oxygène de l'eau interstitielle), ainsi que l'intensité du lessivage des roches, constituent le paramètre déterminant dans la cinétique de vieillissement du minerai de fer, plus que le temps d'exposition à l'atmosphère minière. Le temps d'exposition va, bien sûr, jouer un rôle, de mème que la profondeur dans le pilier et la teneur en calcite de la roche, mais les conditions locales demeurent prépondérantes. A cet égard, le drainage à l'échelle de l'ensemble de la formation 
peut apparaître comme un paramètre important. La teneur en calcite dans le ciment inter-oolitique va déterminer l'intensité du vieillissement de par ses propriétés de tampon acide. Ainsi, dans le faciès très carbonaté du minerai de fer, les carbonates de calcium vont rendre négligeables les néoformations.

Les réactions d'oxydation précédentes peuvent être favorisées dans les milieux riches en fer par l'intervention de bactéries oxydantes du fer (Ferris, 1989). Les conditions climatiques et physico-chimiques régnant dans les mines de fer abandonnées sont propices à l'activité bactérienne. Ces conditions sont : température tempérée et stable (environ $15^{\circ} \mathrm{C}$ ), humidité importante et présence en abondance de fer à l'état ferreux (et donc réduit) dans le minerai de fer. Les bactéries chimiolithotrophes utilisent en effet l'énergie fournie par les réactions d'oxydation, du fer notamment, pour vivre. Par ailleurs, l'activité bactérienne et l'oxydation du fer ferreux sont favorisées par un $\mathrm{pH}$ acide. Dans un milieu globalement tamponné comme le minerai de fer, les bactéries peuvent localement oxyder le fer ferreux en fer ferrique (1) à l'origine de la précipitation d'oxydes ou d'hydroxydes de fer (2). L'acidité nécessaire à l'entretien de ces réactions d'oxydation du fer peut être apportée, comme nous l'avons vu précédemment, par la présence en solution d'une quantité importante de gaz carbonique (4). De la même façon, les bactéries consommatrices de phosphore, contenu dans la goethite formant le cortex oolitique, peuvent expliquer sa dégradation par une désorganisation de sa structure cristalline.

Des échantillons de minerai ont été prélevés dans le stot de Tressange afin de réaliser des analyses bactériologiques :

-prélèvement d'eau de percolation (sous le toit d'une chambre) :

- prélèvement de boues rouges près d'un pilier :

- prélèvement d'échantillons de minerai de fer de la couche grise sur différents piliers correspondant à des dates d'exploitation différentes ;

- prélèvement de l'air minier près de chacun des piliers précédents.

Les incubations sont réalisées en flacons pour sérologie et, pour les cultures anaérobies, le dégagement de $\mathrm{CO}_{\text {est }}$ mesuré. Pour chaque essai, des témoins stérifles sont réalisés. En parallèle, des étalements sur boite de Pétri permettent de dénombrer les microflores (autotrophes ou hétérotrophes) présentes. Après incubation aérobie ou anaérobie $(2$ mois à $24^{\circ} \mathrm{C}$ ), les solutions sont analysées par ICP. Les solutions d'eau de mine et les solides sont également analysés par ICP.

Les résultats de microbiologie sont, pour l'instant, préliminaires. Des microflores hétérotrophes (utilisant le carbone d'origine organique), composées de bactéries, et autotrophes (utilisant le gaz carbonique dissous) ont été isolées des eaux à l'équilibre avec les échantillons solides. Une partie de la microflore hétérotrophe est plutôt oligotrophe et donc particulièrement bien adaptée à croître dans des milieux pauvres en carbone organique. L'implication des bactéries dans la dégradation des matières organiques conduit à la minéralisation (production de $\mathrm{CO}_{2}$ ) d'une partie du carbone organique initialement présent dans l'échantillon. Cette minéralisation faible est observable dans les incubations anaérobies. L'activité altérante des bactéries, visà-vis des phases minérales, est significative dans les échantillons qui correspondent aux roches les moins altérées initialement et peut sans doute conduire à ia formation de la boue rouge riche en fer prélevée dans la mine. On constate effectivement une solubilisation de $\mathrm{Fe}$, Al et Si qui pourrait expliquer la dégradation de la sidérite et de la berthiérine. C'est aussi dans ces échantillons que l'activité minéralisatrice anaérobie est la plus significative. Au vu de ces premiers résultats, le rôle de l'activité bactérienne semble donc primordial. La dégradation de la matière organique, via l'action des bactéries, génère une production de $\mathrm{CO}_{2}$. Ce gaz carbonique permet la libération d'ions $\mathrm{H}^{+}$dans la solution selon la réaction (4) et peut ainsi contribuer au processus d'oxydation. Par ailleurs, les bactéries ont également une activité minéralisatrice altérante vis-à-vis du minerai de fer et cette activité peut être anaérobie.

La sidérite peut présenter des phases superficielles solubilisées riches en fer II. Celles-ci représentent des apports en fer II et constituent donc des substrats ènergétiques et bio-disponibles d'emblée en solution pour les micro-organismes qui les utilisent principalement (Toniazzo, 1998). Thiobacillus ferrooxidans, dans le cas des schistes "carton», peut oxyder directement le fer II, ou indirectement par production en solution d'un oxydant puissant, le fer ferrique (Rakontondratsima, 1995). Le fer II provenant de l'oxydation aqueuse de la sidérite peut également ètre oxydé par voie bactérienne, selon la réaction (1) et le fer III produit peut réagir avec la sidérite et accélérer la dissolution de celle-ci (cycle oxydatif). Si ces conditions idéales persistent, l'oxydation bactérienne de la sidérite peut résulter, comme dans le cas de l'oxydation de la pyrite, de la dissolution du fer maintenu sous forme ferrique par la présence de bactéries oxydatrices du fer. Bien que ces bactéries autotrophes affectionnent particulièrement les milieux acides où le fer est disponible en grande quantité sous forme dissoute, une oxydation à des $\mathrm{pH}$ voisins de la neutralité reste possible. En effet, en présence d'O, dissous ou adsorbé au niveau des micropores, l'action oxydante du fer III peut être maintenue. En revanche, la présence de carbonates limite l'acidification et la solubilité du fer.

Nous avons vu que l'état de fracturation du matériau jouait un rôle primordial dans l'oxydation puisqu'il favorise la présence d'eau et d'oxygène dissous dans l'eau, au contact des minéraux. De mème, les caractéristiques physiques (taille des particules, perméabilité...) des matériaux miniers et le régime hydrologique sont des facteurs qui conditionnent les différentes réactions chimiques (Todd et Reddick, 1997). Les conditions d'oxygénation diffèrent en fonction de l'état de l'exploitation minière. Elles sont au nombre de trois :

- milieu très ventilé : cas de l'aérage actif. La catalyse bactérienne n'est probablement pas critique dans des sections bien ventilées des mines souterraines actives (Kleinmann, 1998). En effet, l'apport des bactéries à l'oxydation du minerai de fer n'est pas primordial bien que la forte concentration d'oxygène dans l'air $\left(9,3 \times 10^{-3}\right.$ mol. $\left.1^{-1}\right)$ leur soit favorable : le processus d'oxydation peut s'engager et se poursuivre sans leur action :

- aérage stoppé : c'est le cas lorsque les travaux miniers prennent fin dans une concession. Dans ces conditions, la concentration d'oxygène diminue de façon significative et l'action des bactéries devient alors primordiale car celles-ci peuvent accélérer fortement la cinétique des réactions d'oxydation ; 
- milieu anaérobie : c'est le cas lorsque les mines sont ennoyées suite à l'arrêt des pompages d'exhaure (fermeture des exploitations). Dans ces conditions, les bactéries aérobies vont avoir une activité tant que l'oxygène disponible sous forme gazeuse dans l'eau n'est pas totalement consommé. Ensuite, l'oxydation et donc l'altération du minerai devraient s'arrêter, à moins qu'un apport d'eau riche en oxygène, via les fractures induites par l'exploitation, puisse parvenir. Globalement, l'eau est renouvelée sauff, peut-être, à certains endroits profonds de la mine où l'oxvdation et donc aussi le vieillissement pourraient prendre fin. Les conditions hydrogéologiques locales deviennent alors un paramètre primordial. Par ailleurs, certaines bactéries, telles que Thiobacillus ferrooxidans, peuvent avoir une activité même en l'absence d'oxygène. Dans ce cas, l'accepteur des électrons libérés par la réaction d'oxydation de fer ferreux devient le fer ferrique en lieu et place de l'oxygène (Corbett et al., 1987). Après ennoyage, l'hypothèse d'une activité bactérienne anaérobie altérante vis-à-vis du minerai de fer est assez probable, d'autant plus que le milieu (c'est-à-dire la mine souterraine) n'est pas clos et donc ouvert à la circulation des bactéries anaérobies.

\section{6}

\section{Influence du vieillissement sur le comportement mécanique du minerai de fer}

L'exposition à l'atmosphère minière des piliers est un paramètre important dans l'évolution du comportement du minerai de fer pour lequel une loi mécanique a été établie (Hoxha et al., 2000). Il faut donc prendre en compte les modifications microstructurales induites par les transformations minéralogiques. Ces dernières s'accompagnent d'un développement de microfissures dans le ciment inter-oolitique et dans les oolites. II s'agit maintenant de traduire ces modifications en terme de variation des propriétés mécaniques. L'altération du ciment, qui est un phénomène micromécanique, se traduit à l'échelle macroscopique par une réduction des différents seuils de la loi de comportement : seuil de plasticité et résistance au pic.

Le faciès ferrifère est le plus sensible au vieillissement. La figure 17 représente les courbes contraintedéformation axiales obtenues à partir d'essais de compression monoaxiale sur la ferri-arénite la plus altérée, exposée à l'atmosphère minière vers 1930, et sur la ferri-arénite saine. On note que la résistance diminue de moitié entre le minerai sain (environ $20 \mathrm{MPa}$ ) et le minerai vieilli (environ $10 \mathrm{MPa}$ ). Par ailleurs, la figure 18 montre les enveloppes des mesures de la résistance au pic de ces deux faciès, obtenues à partir d'une série d'essais triaxiaux à différentes pressions de confinement. La surface au pic est fortement abaissée du fait du vieillissement, ce qui se traduit physiquement par une diminution de la cohésion de la roche. En fait, le minerai de fer a perdu une grande partie de sa cohésion en vieillissant. Pour conclure, le vieillissement du faciès ferrifère du minerai de fer se traduit par une diminution des caractéristiques mécaniques à court terme (diminution de la résistance et du seuil initial de plasticité), sans que les propriétés élastiques (module de Young et coefficient de Poisson) ne soient modifiées.
L'influence sur le comportement mécanique est différente selon le faciès du minerai de fer. Pour le faciès carbonaté, la présence de calcite en abondance réduit les néoformations minéralogiques et les modifications microstructurales, et ceci même si l'échantillon est exposé à l'atmosphère minière depuis près d'un siècle. Aucune modification dans le comportement mécanique n'est constatée par rapport au faciès sain (Grgic, 2001). Le paramètre primordial conditionnant le vieillissement du minerai de fer est donc bien la nature ferrifère du faciès, viennent ensuite les conditions physico-chimiques locales et, finalement, le temps d'exposition à l'atmosphère minière.

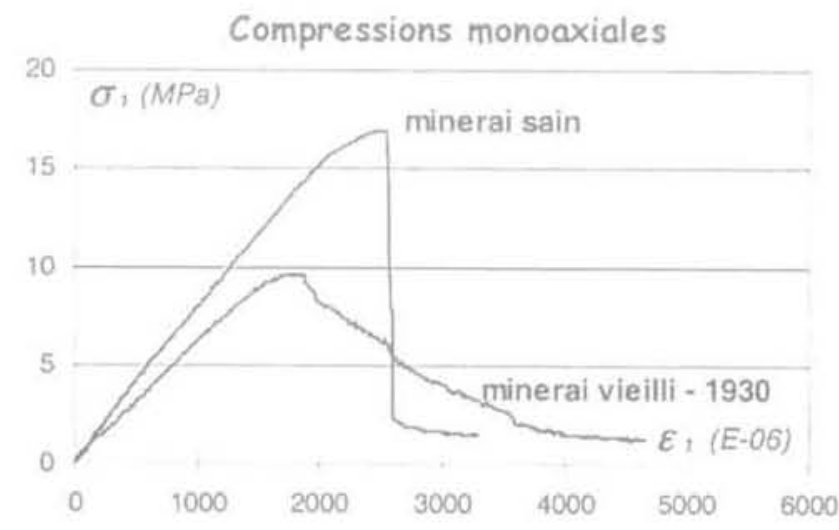

FIG.17 Compressions monoaxiales sur le minerai de fer sain et le minerai de fer vieilli exploité en 1930.

Uniaxial tests on healthy tron ore and on weathered iron ore exploited towards 1930.

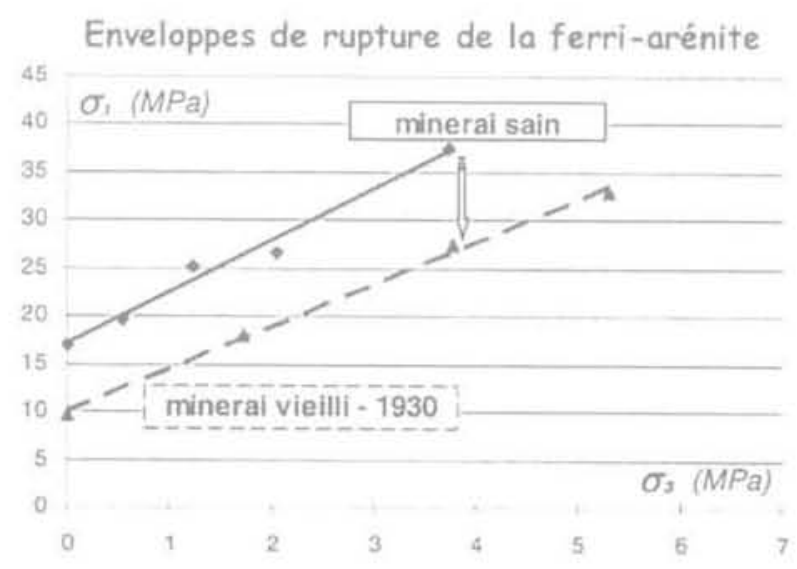

FIG. 18 Enveloppe au pic pour le minerai de fer sain et le minerai de fer vieilli (1930). Peak envelope of healthy iron ore and weathered iron ore (1930). 


\section{Conclusion}

Les mines de fer abandonnées de Lorraine, exploitées selon la méthode des chambres et piliers, posent des problèmes de stabilité dont le vieillissement des roches peut être l'une des causes. L'étude de l'évolution des caractéristiques minéralogiques du minerai de fer lorrain a été réalisée à partir d'échantillons prélevés dans des sondaces carottés dans une zone sans exploitation et dans des piliers abandonnés. Certains sondages, réalisés horizontalement dans des piliers exposés à l'atmosphère minière à différentes époques, sont datés. Les échantillons ont été analysés au MEB, à la microsonde et en diffractométrie X. En parallèle, des analvses microbiologiques, encore en cours, nous ont permis d'étudier l'influence des bactéries sur les phénomènes d'altération des minéraux.

Il résulte de ces études que l'évolution anthropique, c'est-à-dire liée aux travaux miniers, est bien réelle et que ses effets se superposent à l'évolution diagénétique. Cette évolution d'origine anthropique est décrite en deux étapes. La première correspond à une altération physico-chimique du ciment inter-oolitique argileux et carbonaté. Il s'agit d'une hycirolyse des phyllosilicates ferrifères (berthiérines) et d'une oxydation des carbonates de fer (sidérite), La seconde étape correspond dans un premier temps à la transformation et néoformation de berthiérines et dans un deuxième temps à la néoformation d'une croûte d'oxyde de fer qui est finalement un résidu cristallisé à partir des pro- duits libérés au cours de la phase initiale d'altération. Ces transformations minéralogiques sont favorisées par l'activité bactérienne.

La structure interne du minerai est affectée par ces modifications. Certaines oolites sont écrasées, les couches concentriques des cortex intra-oolitiques desquament, la croúte d'oxyde se détache et les oolites n'adhèrent plus à ce résidu de ciment. Ces phénomènes se traduisent à l'échelle macroscopique par une diminution de la cohésion et de la résistance au pic dans des proportions importantes. Ces évolutions d'origine anthropique correspondent à une diagenèse récente ou rétro-diagenèse et peuvent alors être qualifiées de vieillissement dans le sens oủ elles se traduisent par une fragilisation mécanique du minerai de fer. Le vieillissement est donc un paramètre important à prendre en compte dans l'étude de la stabilité à long terme des piliers abandonnés.

Parmi les points restant à élucider, il y a la détermination de l'étendue de la zone vieillie dans les piliers ; des sondages plus profonds $(10 \mathrm{~m})$ sont prévus. En outre, afin de pouvoir réellement prendre en compte l'influence du vieillissement du minerai de fer sur la stabilité à long terme des piliers abandonnés, il faudrait connaitre précisément la cinétique des différentes transformations minéralogiques. Pour cela, l'étude des propriétés physico-chimiques de l'eau interstitielle, par une approche géochimique du problème, est nécessaire pour déterminer les équilibres minéraux initiaux et comprendre l'évolution de ces équilibres dans le temps et en fonction des conditions environnantes.

\section{$\overline{\text { Bibliographie }}$}

Bubenicek L. - $\alpha$ Recherches sur la constitution et la répartition des minerais de fer dans I'Aalènien de Lorraine n. Sciences de la Terre. Nancy 8, 1961, p. 5 204.

Bubenicek L - uGéologie du gisement de fer de Lorraine . Thèse de la faculté des Sciences, Nancy, tome 1, 1970, $146 \mathrm{p}$.

Burchard E. F. - w Iron ore in Cass, Marion. Morris and Cherokee Counties. Texas 1. F.L. Ransome and H.S. Gale, geologist in charge, contributions to economic geology. U.S. geological survey bulletin 620, 1916, p. 69-109

Burkhalter R.M - "Ooidal ironstones and ferruginous microbiolites: origin and relation to sequence stratigraphy (Aalenian and Bajocian, Swiss Jura mountains) 13. Sedimentology 42, 1995, p. 57 74.

Cojan 1., Renard M. - Sédimentologie. Enseignement des sciences de la Terre. Masson, 1997, $418 \mathrm{p}$

Corbett C.M. Ingledew W.J. - $\alpha$ is $\mathrm{Fe}^{3}-\mathrm{k}$. cycling an intermediate in sulphur oxidation by Fe* grown Thiobacillus ferrooxidans? y FEMS microbiology 41. 1987, p. 1-6.

Dagallier G. - «Analyse des carottes des sondages $\mathrm{SN} 1, \mathrm{SN} 2, \mathrm{SN} 3$ réalisés dans la formation ferrifère aalénienne á Joư (54) n. Rapport d'étude, ENSG-INPL. Nancy, $1997.8 \mathrm{p}$.

Deer W.A. Howie R.A., Zussman J. - An Introduction to the Rock Forming Mine rals. Ed. Longmans, $1962,528 \mathrm{p}$.
Ferris F.G., Tazaki K., Fyte W.S. - "Iron oxides in acid mine drainage environ. ments and their association with bacteria i. Chemical geolocy 16, 1989, p. 149. 152.

Grgic D. - «Modélisation du comporte. ment à court et à long terme des roches de la formation ferrifere lorraine. Thèse de doctorat. INPL. Nancy, 2001. $182 \mathrm{p}$.

Kleinmann R.L.P. - "Coal mine Drainage Prediction and pollution Prevention in Perinsylvania s. Chap, 15. Bactericida Control of Acidic Drainage. The Pennsylvania Department of Environmental Protection, oct. 1998, $6 \mathrm{p}$.

Konhauser KO - a Diversity of bacterial iron mineralization 1). Earth-science revjews 43, 1998, p. 91-121.

Loef J.J. - "Composition and genesis of rattlestones from Dutch soils as shown by Mössbauer spectroscopy, INAA and XRD 1. Netherlands journal of geosciences 79 (1), 2000, p. 59-71.

Mustin C. - is Approche physico-chimique et modélisation de l'oxydation bactérienne de la pyrite de Thiobacillus fernooxidans : róle déterminant de la phase minerale n. Thèse de doctorat, Université de Nancy I, 1992.

Pineau J.L. - wContribution à la caractérisation gèomécanique des roches. Application au minerai de fer lorrain w. These de doctorat, INPL, Nancy, 1978, 252 p.

Rakotondratsima LY G - «Mécanismes de gonflement et d'altération des schistes carton n. Thèse de doctorat, INPL. Nancy, 1995, 234 p.

Rose A. W. Cravotta C. A. - « Coal mine Drainage Prediction and pollution Prevention in Pennsylvania s. Chap. 1. Geo chemistry of Coal Mine Drainage. The Pennsylvania Department of Environmental Protection, oct. 1998, 22 p.

Senkavi A.L.. Dixon J.B.. Hossner L.R. (i) Todorokite, goethite and bematite Giteration products of siderite in East Texas lignite overburden 13 . Soil science 142. 1986, p. $36-42$

Teyssen T. - " A depositional model for the liassic Minette ironstones (Luxembourg and France), in comparison with other phanerozoic ooidal ironstones v. Specia publication qeological society London 46. 1989, p. 79-92.

Todd J. Reddick K. - "Groundwater Pollution Primer. Acid Mine Drainage n. Civi Engineering Dept. Virginia Tech. 1997. $5 \mathrm{p}$.

Toniazzo V. - "Approche des mécanismes d'oxvdation de la pyrite par Thiobacillus ferrooxidans gráce à la modélisation morpho-chimique de la surface miné. rale: rôle fondamental des phases superficielles oxydèes $x$. Thèse de doctorat, université Henri-Poincaré, Nancy 1 . 1998, 228p.

Velde B. - «Phyllosilicate formation in berthierine peloids and iron oolites 1 . Special publication geological sociefy London 46, 1989, p. 3-8. 